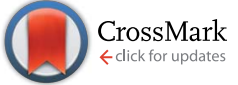

Cite this: Chem. Sci., 2016, 7, 4355

\title{
Benzene-cored AlEgens for deep-blue OLEDs: high performance without hole-transporting layers, and unexpected excellent host for orange emission as a side-effect $\dagger$
}

\author{
Xuejun Zhan,,$^{\mathrm{a}}$ Zhongbin Wu, + $^{\mathrm{b}}$ Yuxuan Lin, ${ }^{\mathrm{a}}$ Yujun Xie, ${ }^{\mathrm{a}}$ Qian Peng, ${ }^{\mathrm{c}}$ Qianqian Li, ${ }^{\mathrm{a}}$ \\ Dongge $\mathrm{Ma}^{* \mathrm{~b}}$ and Zhen $\mathrm{Li}^{* a}$
}

Great efforts have been devoted to explore efficient fluorescent materials, especially deep-blue luminogens, for organic light emitting diodes (OLEDs). In this paper, according to the design idea of creating luminogens with the characteristic of aggregation induced emission (AIE), four new benzenecored luminogens with very simple structure have been intelligently designed, in which, without an additional hole-transporting layer (such as NPB), 3TPA-CN exhibited deep-blue emission and high performance in a simple nondoped LED device with a current efficiency (CE) of $5.21 \mathrm{~cd} \mathrm{~A}^{-1}$, external quantum efficiency (EQE) of $3.89 \%$, and CIE coordinates of $(0.15,0.14)$. Excitingly, as a wonderful sideeffect, 3TPA-CN can serve as an excellent host for orange emissive phosphorescent OLEDs (PhOLEDs), with a maximum current and power efficiency of $57.4 \mathrm{~cd} \mathrm{~A}^{-1}$ and $52.0 \mathrm{~lm} \mathrm{~W}^{-1}$, respectively, and a corresponding maximum EQE of $18.2 \%$, higher than that of CBP $(15.7 \%)$, one popular host for orange PhOLEDs, under the same conditions, thus broadening the utilization of AlEgens as host in PhOLEDs.

Received 4th February 2016
Accepted 16th March 2016
DOI: 10.1039/c6sc00559d
www.rsc.org/chemicalscience

\section{Introduction}

Prompted by both scientific and commercial interests, organic materials with high performance are badly needed for organic light-emitting diodes (OLEDs), including high efficient luminogens and good transporting molecules as the emitting layer, transporting layer or host. ${ }^{1-5}$ Thanks to the great efforts of scientists, many excellent organic molecules have been reported with high OLED performance, especially those with dual functionalities, for example, those acting as emitting and transporting layer simultaneously. ${ }^{6-12}$ Pioneered by Adachi, recent reports of highly efficient thermally activated delayed fluorescent materials, emphasized the importance of pure organic fluorophores again. However, in comparison with their red and green counterparts with satisfactory efficiencies, good blue organic light-emitting materials were still scarce, mainly due to the intrinsic large band gap and unwanted aggregation-caused

\footnotetext{
${ }^{a}$ Department of Chemistry, Hubei Key Lab on Organic and Polymeric Opto-Electronic Materials, Wuhan University, Wuhan, 430072, China. E-mail: lizhen@whu.edu.cn; lichemlab@163.com; Fax: +86-27-68756757; Tel: +86-27-68755363

${ }^{b}$ Changchun Institute of Applied Chemistry, The Chinese Academy of Sciences, Changchun, 130022, China. E-mail: mdg1014@ciac.jl.cn

'Institute of Chemistry, The Chinese Academy of Sciences, Beijing 100190, China

$\dagger$ Electronic supplementary information (ESI) available: Synthetic procedures, TGA and DSC curves, PL spectra, XRD curves and detailed device performances. See DOI: 10.1039/c6sc00559d

$\ddagger$ The authors contributed equally to this work.
}

quenching (ACQ) effect caused by the possible formation of excimers. ${ }^{13-17}$ Fortunately, since the first report of Ben Zhong Tang in 2001, a new type of luminogen, AIEgens, with a propeller-like structure, has emerged and become a hot research topic, which emitted weakly in solution but much stronger in the aggregation state (termed as aggregation induced emission, AIE), derived from the restricted intramolecular motion (RIM) of some aromatic rings. ${ }^{18-22}$ Regardless of the huge family of AIEgens with different emission color developed so far, according to the RIM mechanism, there have been relatively few reports concerning blue and deep-blue AIEgens. ${ }^{23-27}$

Recently, by tuning the balance of the intramolecular motion and conjugation through different linkage mode or/ and adjusting twist angles between the aromatic construction blocks, we have successfully obtained some blue and deepblue AIEgens (Chart S1, ESI $\dagger$ ). Among them, TPA-CN-TPA ${ }^{28}$ with a structural platform of polyphenylbenzene, exhibited a high OLED performance (4.5 cd $\left.\mathrm{A}^{-1}\right)$ and deep-blue emission $\left(\lambda_{\mathrm{EL}}=431 \mathrm{~nm}\right)$. To simplify the tedious synthesis of TPA-CN-TPA (Chart S2, ESI $\dagger$ ), and more importantly, endow it with multi-functionalities, the rational molecular design is still required. As the transporting property of lightemitting materials contributes much to the corresponding LED performance, this function becomes the first choice, which, generally, is also the key parameter for the host materials..$^{29-31}$ 
Prompted by all the above considerations, two luminogens, 2TPA-CN and 3TPA-CN, with very simple structures constructed of only phenyl and triphenylamino groups, were designed and synthesized conveniently. For comparison, two tetraphenylethene (TPE)-based analogues of 2TPE-CN and 3TPE-CN were also prepared (Scheme 1). Excitingly, without the required holetransporting layer (such as NPB: $N, N^{\prime}$-bis-(1-naphthyl)- $N, N^{\prime}$ biphenyl-1,1'-biphenyl-4,4'-diamine) for a standard LED device, the simple nondoped LED devices containing 3TPA-CN as emitting layer showed high performance. The maximum current efficiency (CE) and external quantum efficiency (EQE) were $5.21 \mathrm{~cd} \mathrm{~A}^{-1}$ and $3.89 \%$, respectively, while the CIE coordinates were $(0.15,0.14)$. More importantly, thanks to its good hole-transporting ability, the device with 3TPA-CN as host could achieve excellent LED performance, with a maximum current and power efficiency of $57.4 \mathrm{~cd} \mathrm{~A}^{-1}$ and $52.0 \mathrm{~lm} \mathrm{~W}^{-1}$, respectively, and a corresponding maximum EQE of $18.2 \%$, higher than those of the generally used host of 4,4'-bis( $9 H$-carbazol-9yl)biphenyl (CBP) under the same conditions. To the best of our knowledge, this is the first time that AIEgens have been successfully utilized as hosts in PhOLEDs. Herein, we would like to present the synthesis, AIE characteristics, thermal, photophysical, electrochemical and electroluminescent (EL) properties in detail.

\section{Results and discussion}

\section{Synthesis}

The four benzene-cored luminogens (Scheme 1) were obtained similarly through two synthetic steps with good yields (Scheme $\mathrm{S} 1, \mathrm{ESI} \dagger)$. The key intermediates of 2,6-dibromobenzonitrile and 2,4,6-tribromobenzonitrile were synthesized when the corresponding amines were treated with $\mathrm{CuCN}$ and $t$-BuONO. Finally, through a palladium (0)-catalyzed Suzuki crosscoupling reaction between 2,6-dibromobenzonitrile/2,4,6-tribromobenzonitrile and 4-Bpin-tetraphenylethene/4-Bpin-triphenylamine, 2TPA-CN, 3TPA-CN, 2TPE-CN and 3TPE-CN could be obtained with good yields.

All these new compounds were purified by column chromatography on silica gel using petroleum ether-chloroform as eluent and fully characterized by ${ }^{1} \mathrm{H}$ and ${ }^{13} \mathrm{C}$ NMR, mass spectrometry, and elemental analysis.

\section{Thermal properties}

Generally, the good thermal stability of the luminogens can be beneficial to the process of vacuum deposition and operating stability of OLED devices. The thermal properties of all these new emitters were investigated by thermogravimetric analysis (TGA) and differential scanning calorimetry (DSC) measurement. As depicted in Fig. 1 and Table 1, these luminogens are thermally stable with $T_{\mathrm{d}}$ values (corresponding to $5 \%$ weight loss) in the range of $377-480{ }^{\circ} \mathrm{C}$. Owing to the good thermal stability of tetraphenylethene, compounds with tetraphenylethene units (2TPE-CN and 3TPE-CN) show better thermal stability than those containing TPA units (2TPA-CN and 3TPACN). The decomposition temperatures of the threefold Suzuki products are higher when compared to the twofold ones. The glass transition temperatures $\left(T_{\mathrm{g}}\right)$ of 3TPA-CN and 3TPE-CN are 126 and $156{ }^{\circ} \mathrm{C}$, respectively, further demonstrating better thermal stability of 3TPA-CN and 3TPE-CN (Fig. S1, ESI $\dagger$ ). Thus, the good thermal properties would contribute to the good performance of their corresponding LED devices.

\section{Photophysical properties}

All these compounds have fine to good solubility in common organic solvents, such as dichloromethane, chloroform and tetrahydrofuran (THF) etc. Fig. 2 shows their absorption spectra in diluted THF solutions. In the solutions, 2TPA-CN and 3TPA-CN demonstrate similar absorptions, in which the bands at about 300 and $360 \mathrm{~nm}$ can be ascribed to the triphenylamine-centered $\mathrm{n}-\pi^{*}$ transition and intramolecular charge transfer (ICT) transition (Fig. S2, ESI $\dagger$ ), respectively. Analyzing the absorbance carefully, when decorated with three TPA units, due to the enhanced electron donating ability, the intramolecular charge transfer (ICT) transition of 3TPA-CN is enhanced in comparison with that bearing two TPA ones. 2TPE-CN and 3TPE-CN also exhibit similar absorptions, the bands around $330 \mathrm{~nm}$ could be attributed to the $\pi-\pi *$ transition, in which the ICT transition is weak. This can be
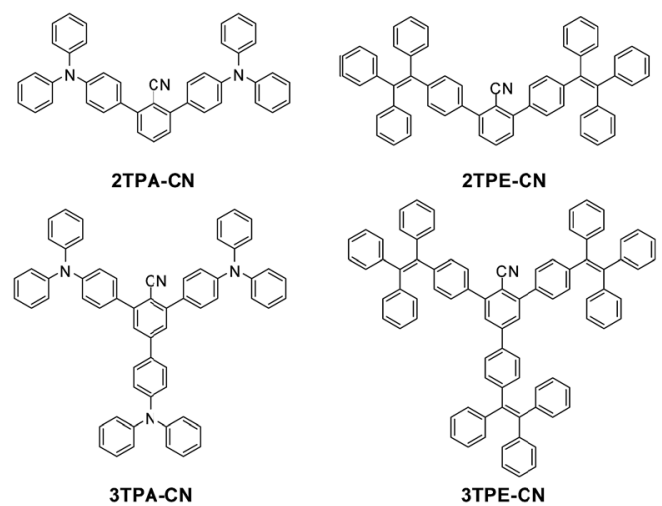

Scheme 1 Chemical structures of the synthesized luminogens.

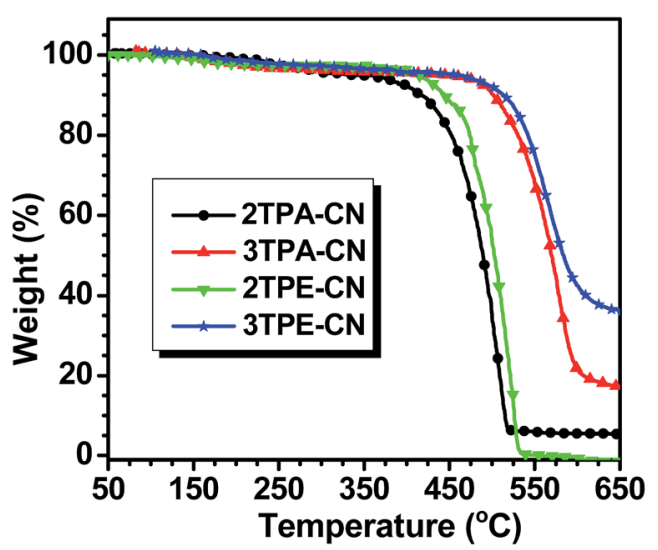

Fig. 1 TGA curves recorded under $\mathrm{N}_{2}$ at a heating rate of $10^{\circ} \mathrm{C} \mathrm{min}^{-1}$. 
Table 1 The thermal, electrochemical and photophysical data of the luminogens

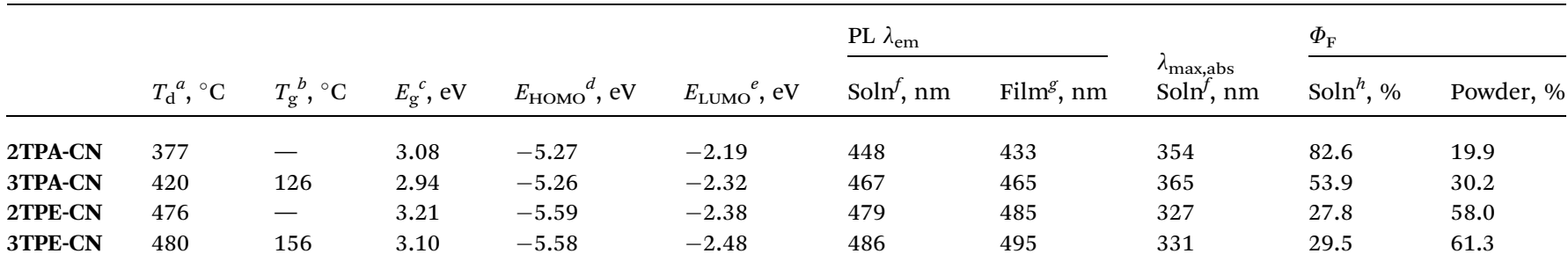

${ }^{a} 5 \%$ weight loss temperature measured by TGA under $\mathrm{N}_{2} \cdot{ }^{b}$ Glass-transition temperature measured by DSC under $\mathrm{N}_{2} \cdot{ }^{c}$ Band gap estimated from optical absorption band edge of the solution. ${ }^{d}$ Calculated from the onset oxidation potentials of the compounds. ${ }^{e}$ Estimated using empirical equations $E_{\mathrm{LUMO}}=E_{\mathrm{HOMO}}+E_{\mathrm{g} .}{ }^{f}$ Determined in THF solution (2TPA-CN and 3TPA-CN) and THF/ $\mathrm{H}_{2} \mathrm{O}$ (5:95) (2TPE-CN and 3TPE-CN), respectively. ${ }^{g}$ On glass. ${ }^{h}$ Observed from absorption spectra in dilute THF solution, $10 \mu \mathrm{M}$.
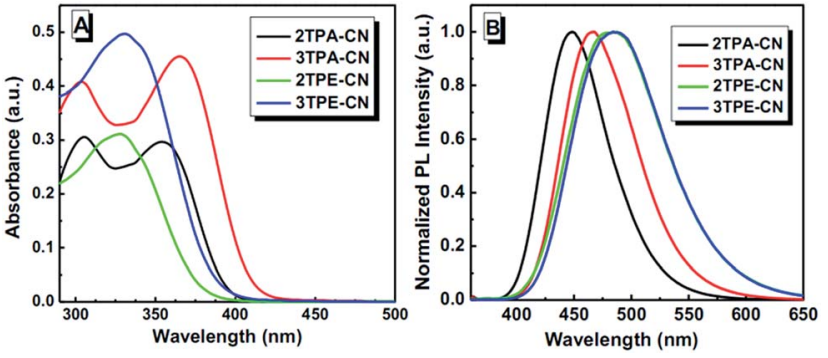

Fig. 2 UV-vis (A) and PL (B) spectra of the four emitters in THF solutions.

evidenced from the calculated UV-vis spectra of TPE based luminogens: the fraction of HOMO-LUMO transition is weak along with a relatively small oscillator strength (Fig. S4 and S5 and Tables S1 and S2, ESI $\dagger$ ).

In the PL spectra (Fig. 2B), their emissions in diluted solutions are mainly located at $445-490 \mathrm{~nm}$. According to the conjugation effect and inductive effect, once the para-position of cyano group was substituted by TPA or TPE units, the intramolecular ICT effect should be enhanced to a large degree. Thus, as a result of the enhanced electron donating ability and increased conjugation, the emission wavelengths of 3TPA-CN $(467 \mathrm{~nm})$ and 3TPE-CN $(486 \mathrm{~nm})$ are red-shifted 19 and $7 \mathrm{~nm}$, in comparison with those of 2TPA-CN (448 nm) and 2TPE-CN (479 $\mathrm{nm}$ ), respectively. Fortunately, the emissions are still in the blue range, partially due to their twist conformation.

In our previous study, owing to the twisted conformation, the emission wavelength of Ph3TPE was $494 \mathrm{~nm}$, while that of Ph2TPE was $498 \mathrm{~nm}$, even though the number of periphery TPE units had increased (Chart S3, ESI $†$ ). ${ }^{32}$ However, here, once the alkoxy group was altered to the electron withdrawing cyano group, a red shifted emission wavelength of 3TPE-CN was observed compared to that of 2TPE-CN. This is reasonable. The ICT effect plays an important role even it is relatively weaker than that in TPA-based luminogens.

We further investigated the PL properties of the luminogens in the solid state, as materials are often utilized in the solid state in practical applications. As shown in Table 1, the four derivatives show emissions ranging from $433 \mathrm{~nm}$ to $495 \mathrm{~nm}$. In the PL spectra of their thin films, TPA-based luminogens exhibit much narrow emissions with fwhm (full width at halfmaximum) of about $50 \mathrm{~nm}$. 2TPA-CN exhibits a relatively large blue shifted emission of $433 \mathrm{~nm}$ in the solid state, compared to that in solution. This is beneficial to achieving good performance of blue OLEDs. A similar phenomenon can be observed in 3TPA-CN, regardless of the small blue shift of $2 \mathrm{~nm}$. This is reasonable when taking the ICT effect into account: although a twist structure like 2TPA-CN can induce a relatively large blue shifted emission, the larger dipolar effect in 3TPA-CN can cause a converse effect, that is, red-shifted emission. What we observed is the net result of the above two conflicting effects. However, unlike TPA-based luminogens with blue-shifted emissions in films (compared to those in solutions), the two TPE-based emitters exhibit red-shifted emissions in the solid state, caused by the restricted rotation of the aromatic rings in TPE and the following increased conjugation, similar to other reported AIEgens. However, thanks to their twist structures, all the emissions are shorter than $500 \mathrm{~nm}$, which is well consistent with our design idea.

To quantitatively know more about the fluorescent properties of these luminogens, their fluorescent quantum yields (QY) were also estimated (Table 1), as referenced to 9,10-diphenylanthracene ( $\Phi_{\mathrm{F}}=0.91$ in hexane). The fluorescent quantum yields of TPA-based products surpass $50 \%$, owing to the well modified $\mathrm{D}-\pi-\mathrm{A}$ molecular structures, which could provide highly emissive ICT excited states upon excitation. ${ }^{33}$ For TPEbased luminogens, due to their highly twisted conformations and weak ICT effect, lower quantum yields were found in comparison with those of TPA-based luminogens, but still higher than similar AIEgens without a cyano group.

\section{Aggregation induced emission (AIE) and aggregation enhanced emission (AEE) properties}

Considering the structural characteristic and existence of TPE, in order to study the possible AEE or AIE characteristic of these luminogens, we chose cyclohexane $/ \mathrm{H}_{2} \mathrm{O}$ and THF as the solvent pair for their miscibility and recorded the photoluminescence (PL) change. Fig. S7† shows the PL spectra of TPA-based luminogens in THF/cyclohexane mixtures with different cyclohexane fractions $\left(f_{\mathrm{w}}\right)$, in which the solvent polarity and extent of solute aggregation could be well tuned. Taking 3TPA-CN as an example, with the gradual addition of cyclohexane, the PL 
intensities increase when the cyclohexane content is higher than $20 \%$. Further increasing the cyclohexane content to $99 \%$, the emission intensity of 3TPA-CN still increases. As shown in Fig. S8, $\uparrow$ when the cyclohexane fraction is $20 \%$, the QY increases. This trend is maintained up to $50 \%$. At higher fractions of cyclohexane, due to the poor solubility of the luminogens, the percentage of molecules inside the nanoparticles (which do not contribute to the QY) increases, ${ }^{28}$ which decreases the obtained quantum yield directly. Further increasing the cyclohexane fractions, the QYs fluctuate. According to these results, these TPA-based luminogens show weak AEE characteristics or at least resistant to the ACQ effect. Moreover, the PL peaks blue-shift when the cyclohexane content increases, probably due to the solvation effect (Fig. S2, ESI $\dagger$ ) or/and the morphological change of the aggregates from the amorphous to crystalline state. This is beneficial to the fabrication of deepblue light-emitting devices. Similar to other TPE-containing AIEgens, 2TPE-CN and 3TPE-CN show typical AIE phenomena. Taking 3TPE-CN as an example, in dilute THF solution, the PL spectrum is almost a flat line parallel to the abscissa, indicating that it is nearly nonemissive in the solution state. However, when a large amount of water is added, intense emissions can be observed (Fig. 3). When the water fraction is over $60 \%$, the PL intensity increases swiftly, due to the formation of aggregates. At a $f_{\mathrm{w}}$ value of $95 \%$, the PL intensity is the highest one with a peak located at $486 \mathrm{~nm}$ for 3TPE-CN, which is about 300 -fold higher than that in pure THF.

A similar phenomenon could also be observed for 2TPE-CN (Fig. S9, ESI $\dagger$ ). From pure THF solution to aggregate states in a mixture of solvents of THF and water with the ratio of $1: 99$, the $\Phi_{\mathrm{F}}$ values of 2TPE-CN and 3TPE-CN increase from $0.11 \%$ and $0.13 \%$ to $38.8 \%$ and $27.4 \%$, respectively, further confirming their AIE activity.

Thermally activated delayed fluorescence (TADF) can lead to possible $100 \%$ internal singlet yields through the up-conversion of triplet to singlet states. ${ }^{5,33,34}$ Considering the twisted structure and the existence of intramolecular charge-transfer (ICT) state, we also tested the low-temperature fluorescence and
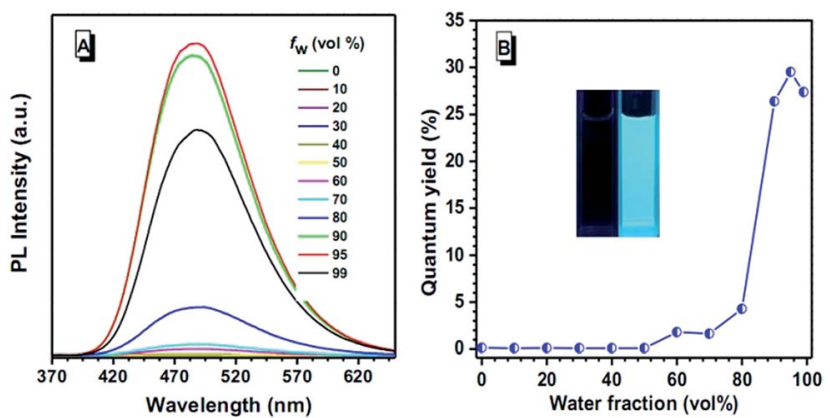

Fig. 3 (A) PL spectra of 3TPE-CN in THF/ $\mathrm{H}_{2} \mathrm{O}$ mixtures with different water fractions $\left(f_{\mathrm{w}}\right)$. (B) Plots of fluorescence quantum yields determined in THF/ $\mathrm{H}_{2} \mathrm{O}$ solutions using 9,10-diphenylanthracene $(\Phi=90 \%$ in cyclohexane) as standard versus water fractions. Inset in (B): photos of 3TPE-CN in THF/water mixtures $\left(f_{\mathrm{w}}=0\right.$ and $\left.99 \%\right)$ taken under the illumination of a $365 \mathrm{~nm}$ UV lamp. UV-vis (A) and PL (B) spectra of the four emitters in THF solutions. phosphorescence (Fig. S10, ESI $\dagger$ ). The phosphorescent spectra of TPE-based luminogens are too weak. For 2TPA-CN and 3TPA$\mathbf{C N}$, their $\Delta E_{\mathrm{ST}}$ values are calculated to be $0.45 \mathrm{eV}$ and $0.48 \mathrm{eV}$, respectively. Typically, the energy distance $\left(\Delta E_{\mathrm{ST}}\right)$ between the lowest singlet state and the lowest triplet state should be small enough. ${ }^{5}$ However, in our case, these $\Delta E_{\mathrm{ST}}$ values are not so small as to guarantee the intersystem crossing (ISC) process. The absence of TADF can also be verified from the life-time test (Fig. S11, ESI $\dagger$ ).

\section{Electrochemical properties and theoretical calculations}

Cyclic voltammetry (CV) measurements were carried out to investigate the electrochemical properties of these luminogens. The highest occupied molecular orbital (HOMO) energy levels were estimated from the onset oxidation potentials according to the equation of HOMO $=-\left(4.8+E_{\mathrm{ox}}\right) \mathrm{eV}$, while the lowest unoccupied molecular orbital (LUMO) energy levels were obtained from optical band-gap energies (estimated from the onset wavelengths of the UV absorptions) and HOMO values. As shown in Table 1, for 2TPA-CN, 3TPA-CN, 2TPE-CN and 3TPE$\mathbf{C N}$, their HOMO values are calculated to be $-5.27,-5.26,-5.59$ and $-5.58 \mathrm{eV}$, respectively. The HOMO levels of TPA-based luminogens are a little higher than that of NPB $(-5.30 \mathrm{eV})$, thus, better device performance could be expected, partially due to the relatively easier transfer of holes and the resultant lower turn-on voltages. ${ }^{17}$ Similar to other AIEgens, TPE-based luminogens show deeper HOMO levels, indicating their better stability to resistant oxidation of the environment. Their corresponding LUMO energy levels are calculated to be -2.19 , $-2.32,-2.38$ and $-2.48 \mathrm{eV}$, respectively, showing a consecutive change. As the LUMO level of traditional electron-transport material TPBi is about $-2.7 \mathrm{eV}$, the transfer of electrons will be easy in the corresponding OLED devices. Moreover, the large band-gaps of 2TPE-CN (3.21 eV) and 3TPE-CN $(3.10 \mathrm{eV})$ compared to those of TPA-based emitters, indicate their relatively poor conjugation and weak ICT effect, owing to the more twisted conformation and weak electron-donating ability of TPE units.

To further explore the structure-property relationship, we have carried out Density Functional Theory (DFT) calculations (B3LYP/6-319*) to obtain the optimized structures and orbital distributions of HOMO and LUMO energy levels of these luminogens. As demonstrated in Fig. 4, the electron clouds of HOMO energy levels are all mainly located at the peripheral TPA or TPE units, due to their electron-donating abilities. The LUMOs of the four luminogens are dominated by orbitals from the central cyano substituted benzene. And there is an obvious overlap of the HOMO and LUMO, which should benefit the transfer of both holes and electrons. In the optimized molecular structures of these four luminogens, it is clearly seen that the intramolecular twist angles are larger in TPE-based luminogens rather than those of TPA-based ones. Also, the introduced cyano group can make the molecules twisted. Taking 3TPA-CN as an example, the twist angle between the TPA unit (para position of the cyano group) and the central benzene is $35.10^{\circ}$, much smaller than that between TPA unit (ortho position of the cyano 


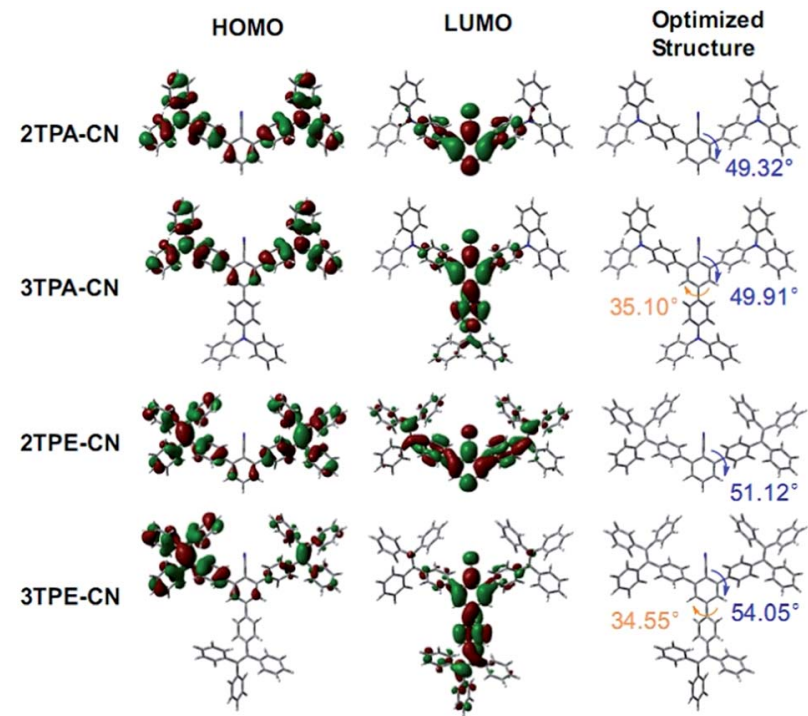

Fig. 4 Calculated molecular orbital amplitude plots of HOMO and LUMO levels and optimized molecular structures.

group) and the central benzene $\left(49.91^{\circ}\right)$. Furthermore, due to the decoration of the third TPA or TPE unit, the twist angle between cyano group and ortho TPA or TPE unit becomes larger in 3TPA-CN or 3TPE-CN in comparison with that in 2TPA-CN or 2TPE-CN, respectively. Just because of the twisted conformations of these luminogens, the conjugation effect is largely decreased between the peripheral unit and the phenyl core, directly leading to the weak ICT effect. This can suppress the red-shifted emission to some degree, and make the blue emission possible. Besides, the predicted values of band-gaps for 2TPA-CN, 3TPA-CN, 2TPE-CN and 3TPE-CN were calculated to be $3.63,3.42,3.79$ and $3.54 \mathrm{eV}$, respectively, which are about $0.5 \mathrm{eV}$ bigger than their optical band-gaps.

\section{Characterization of fluorescent OLEDs}

Considering the hole-transport ability of TPA and TPE units, we first fabricated hole-only devices with the configuration of ITO/ $\mathrm{MoO}_{3}(10 \mathrm{~nm}) / \mathrm{X}(15 \mathrm{~nm}) / \mathrm{MoO}_{3}(10 \mathrm{~nm}) / \mathrm{Al}$ (Fig. 5A), in which, $\mathrm{MoO}_{3}$ worked as the hole-injection and electron-blocking layers and $\mathrm{X}$ refers to 2TPA-CN, 3TPA-CN, 2TPE-CN or 3TPE-CN. Fig. 6

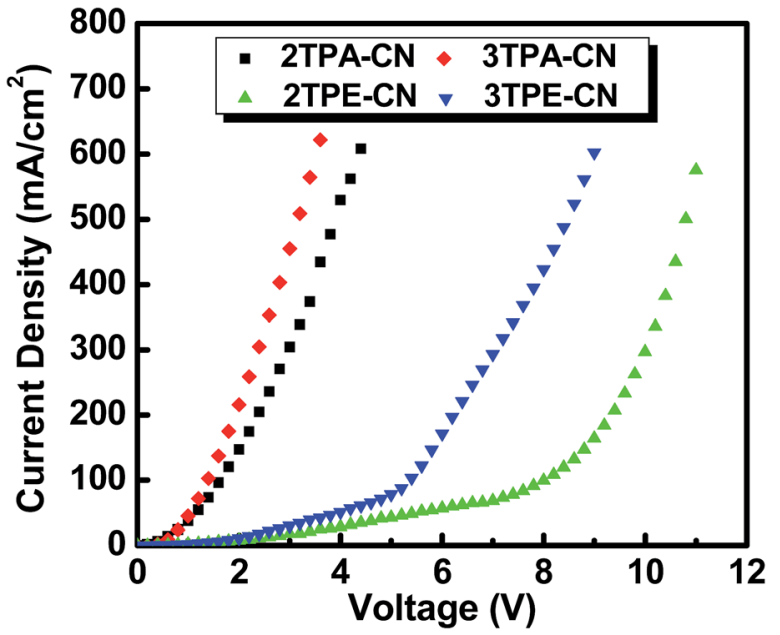

Fig. 6 Current density-voltage characteristics of hole-only devices of the four luminogens.

shows the current density-voltage $(J-V)$ characteristics, and the results confirmed the fine to good hole-transport ability of these luminogens. Due to the better electron-donating ability of TPA units, TPA-based luminogens demonstrate better transport ability in comparison with TPE-based ones. With more TPE units decorated outside the benzene core, 3TPE-CN exhibits better transport ability than 2TPE-CN. Similarly, 3TPA-CN exhibits better transport ability than 2TPA-CN.

To investigate the EL performance of these new luminogens, nondoped fluorescent OLEDs were fabricated with a configuration of ITO/ $/ \mathrm{MoO}_{3}(10 \mathrm{~nm}) / \mathrm{NPB}(60 \mathrm{~nm}) / \mathrm{mCP}(15 \mathrm{~nm}) / \mathrm{X}(30 \mathrm{~nm}) /$ TPBi $(30 \mathrm{~nm}) / \mathrm{LiF}(1 \mathrm{~nm}) / \mathrm{Al}$ (Fig. 5B), in which $\mathrm{MoO}_{3}$, NPB, and TPBi (1,3,5-tri(1-phenyl-1 $H$-benzo[d]imidazol-2-yl)phenyl) worked as the hole-injection, hole-transporting, and electrontransporting/hole-blocking layers, respectively. Otherwise, mCP with a wide band-gap and high triplet state energy $\left(E_{\mathrm{T}}\right)$ can function as the blocking layer to confine excitons in the EML. ${ }^{16}$ Performances of all these devices are shown in Fig. 7 and Table 2. Devices based on TPA-containing luminogens possess deepblue emissions while those of TPE-containing compounds exhibit blue-green ones. Devices based on 3TPE/TPA-containing emitters turn on at lower voltages than those based on 2TPE/ TPA-containing ones, indicating their smaller injection barriers

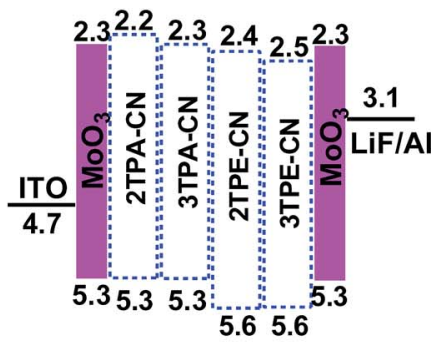

A
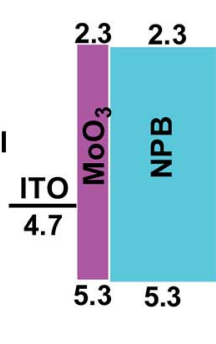

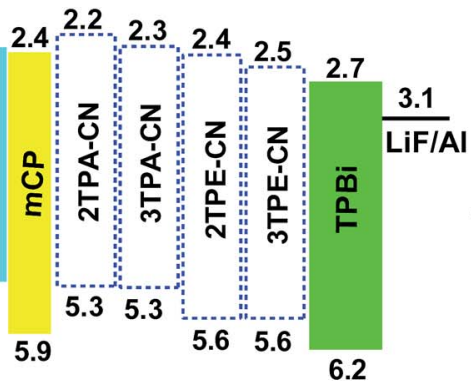

B

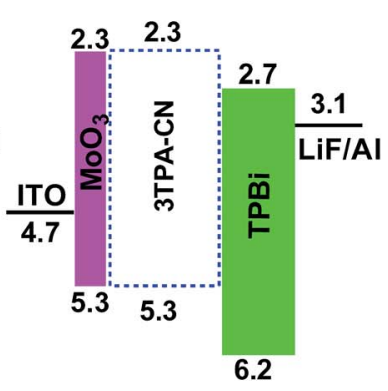

C

Fig. 5 Energy level diagram of the single carrier device (A), traditional non-doped device (B) and device with simple structure (C). ${ }^{35-38}$ 

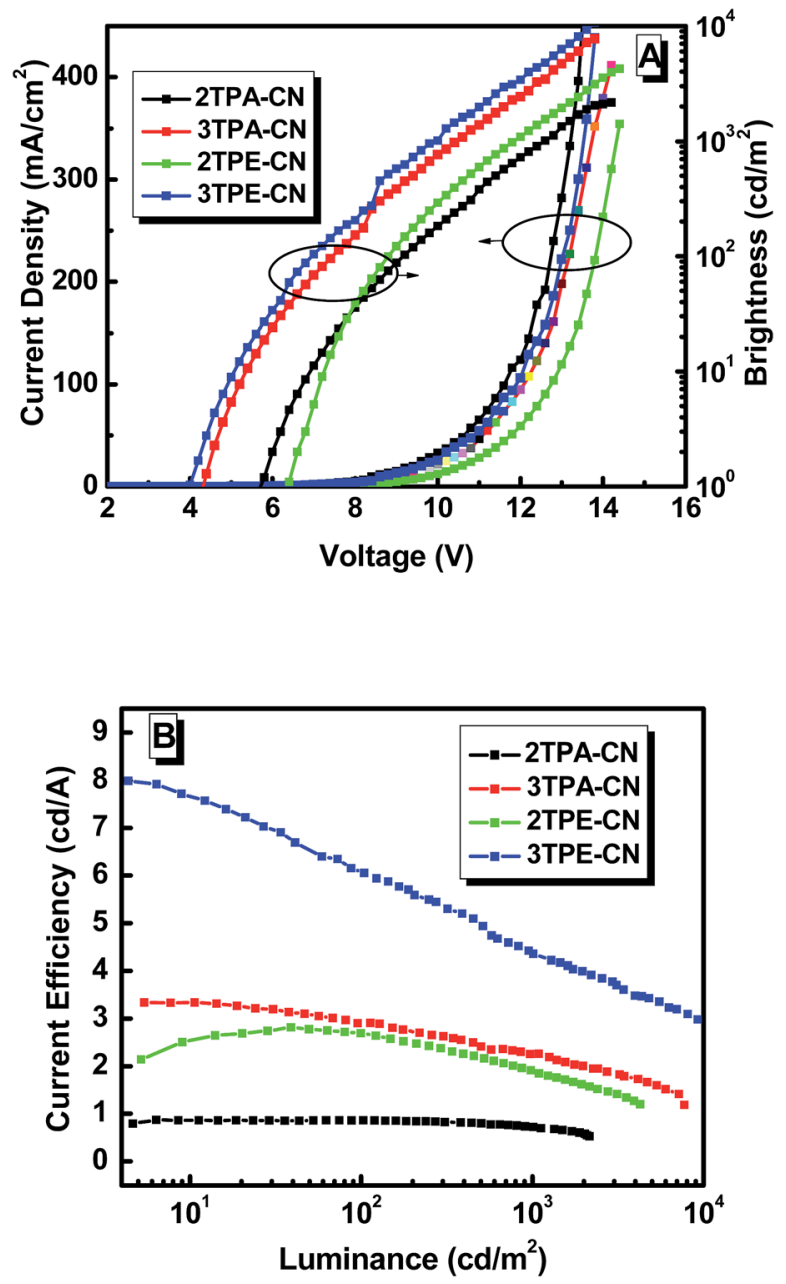

Fig. 7 (A) Current density-voltage-luminance characteristics and (B) current efficiency-luminance characteristics of non-doped devices. between transporting layers and emitters. Considering the similar energy levels of 2TPA-CN and 3TPA-CN (2TPE-CN and 3TPE-CN), the different turn-on voltages can be ascribed to their different transport properties. Besides the lower $V_{\text {on }}$, the device based on 3TPA-CN or 3TPE-CN also shows much higher luminance than that of 2TPA-CN or 2TPE-CN, respectively. The device based on 3TPE-CN exhibits the highest efficiencies with a maximum EQE of $3.06 \%$, CE of $8.03 \mathrm{~cd} \mathrm{~A}^{-1}$, and PE of $5.51 \mathrm{~lm}$ $\mathrm{W}^{-1}$, while that of 3TPA-CN shows relatively good efficiency with maximum EQE of $2.34 \%, \mathrm{CE}$ of $3.34 \mathrm{~cd} \mathrm{~A}^{-1}$, and PE of 2.20 $\operatorname{lm} \mathrm{W}^{-1}$. Meanwhile, the CIE coordinates of $(0.16,0.16)$, in the range of deep blue, is realized. Also, 2TPA-CN-based device possess deep-blue emission with CIE coordinates of $(0.15,0.10)$, closer to the standard deep blue, however, the low CE of $0.88 \mathrm{~cd}$ $\mathrm{A}^{-1}$ indicates that the molecular structure still needs to be optimized. As shown in Fig. S14 (ESI $\dagger$ ), their EL spectra are almost identical at different driving voltages, showing the stability of these luminogens as the emitting material layer (EML).

The above device structure consists of a hole-transporting layer (HTL), an emitting layer, and electron-transporting layers (ETLs). Typically, an OLED device can be simplified if the emitting materials also possess carrier-transporting characteristics. ${ }^{31}$ Based on the good hole transport property, we wonder whether the NPB layer could be removed, and devices with a configuration of ITO/ $\mathrm{MoO}_{3}(10 \mathrm{~nm}) / 3$ TPA-CN $(70 \mathrm{~nm}) / \mathrm{TPBi}(30$ $\mathrm{nm}) / \mathrm{LiF}(1 \mathrm{~nm}) / \mathrm{Al}$ (Fig. 5C) were also fabricated. As shown in Fig. 8 and S15 (ESI $\dagger$ ), 3TPA-CN exhibits much better efficiencies with a $\mathrm{CE}$ of $5.21 \mathrm{~cd} \mathrm{~A}^{-1}$, PE of $5.06 \mathrm{~lm} \mathrm{~W}^{-1}$ and EQE of $3.89 \%$. Notably, when the NPB layer is replaced by the emitting layer, $\lambda_{\mathrm{EL}}$ is blue-shifted to $461 \mathrm{~nm}$ with a deep-blue emission of $(0.15$, 0.14). The maximum luminance is as high as $12363 \mathrm{~cd} \mathrm{~m}^{-2}$. Even at the luminance of $10000 \mathrm{~cd} \mathrm{~m}^{-2}$, the $\mathrm{CE}$ of the device can still remain $2.76 \mathrm{~cd} \mathrm{~A}^{-1}$, demonstrating a relatively low efficiency roll-off. These results can further confirm that 3TPACN retains good hole-transport property, and the use of NPB as an additional hole-transport layer is not necessary, because it

Table 2 EL performances of 2TPA-CN ${ }^{a}, 3 T P A-C N, 2 T P E-C N$ and 3TPE-CN

\begin{tabular}{|c|c|c|c|c|c|c|c|}
\hline Device & $\lambda_{\mathrm{EL}}(\mathrm{nm})$ & $V_{\text {on }}(\mathrm{V})$ & $L_{\max }\left(\mathrm{cd} \mathrm{m}^{-2}\right)$ & $\eta_{\mathrm{P}, \max }\left(\operatorname{lm} \mathrm{w}^{-1}\right)$ & $\eta_{\mathrm{C}, \max }\left(\mathrm{cd} \mathrm{\textrm {A } ^ { - 1 }}\right)$ & $\eta_{\text {ext,max }}(\%)$ & $\operatorname{CIE}^{c}(x, y)$ \\
\hline $\mathrm{A}$ & 445 & 6.0 & 2176 & 0.42 & 0.88 & 0.66 & $0.15,0.10$ \\
\hline B & 466 & 4.4 & 7719 & 2.20 & 3.34 & 2.34 & $0.16,0.16$ \\
\hline $\mathrm{C}$ & 505 & 6.6 & 4279 & 1.13 & 2.82 & 1.07 & $0.21,0.41$ \\
\hline $\mathrm{D}$ & 499 & 4.0 & 10666 & 5.51 & 8.03 & 3.06 & $0.20,0.40$ \\
\hline $\mathrm{E}$ & 461 & 3.0 & 12363 & 5.06 & 5.21 & 3.89 & $0.15,0.14$ \\
\hline $\mathrm{F}$ & 470 & 3.6 & 7257 & 5.02 & 5.04 & 3.35 & $0.15,0.17$ \\
\hline G & 471 & 3.6 & 5906 & 5.06 & 5.07 & 3.27 & $0.14,0.17$ \\
\hline $\mathrm{H}$ & 470 & 3.6 & 9345 & 4.52 & 4.57 & 2.63 & $0.15,0.19$ \\
\hline I & 564 & 2.8 & 48434 & 52.0 & 57.4 & 18.2 & $0.50,0.50$ \\
\hline $\mathrm{J}$ & 562 & 3.2 & 47567 & 41.7 & 50.6 & 15.7 & $0.49,0.51$ \\
\hline
\end{tabular}

${ }^{a}$ Compound and device pairings are as follows: 2TPA-CN (A) ${ }^{b}$, 3TPA-CN (B, E-I), 2TPE-CN (C) and 3TPE-CN (D). ${ }^{b}$ Device configuration: ITO/MoO 3 $(10 \mathrm{~nm}) / \mathrm{X} / \mathrm{TPBi}(30 \mathrm{~nm}) / \mathrm{LiF}(1 \mathrm{~nm}) / \mathrm{Al}$. For device A-D: X = NPB $(60 \mathrm{~nm}) / \mathrm{mCP}(15 \mathrm{~nm}) / \mathrm{emitter}(30 \mathrm{~nm})$. For device E: X = 3TPA-CN $(70 \mathrm{~nm})$. For device $\mathrm{F}-\mathrm{H}: \mathrm{X}=\mathrm{NPB}(60 \mathrm{~nm}) / \mathrm{mCP}(10 \mathrm{~nm}) / \mathrm{BmPyPb}: x \%$ 3TPA-CN $(20 \mathrm{~nm}) / \mathrm{BmPyPb}(10 \mathrm{~nm}), x=30 \%(\mathrm{~F}), 40 \%(\mathrm{G})$ and $50 \%(\mathrm{H})$. For device I-J: $\mathrm{X}=\mathrm{NPB}(60 \mathrm{~nm}) / \mathrm{mCP}(10 \mathrm{~nm}) /$ host:PO-01 $(20 \mathrm{~nm}, 10 \mathrm{wt} \%)$, host $=3$ TPA-CN $(\mathrm{I})$ and CBP $(\mathrm{J}) .{ }^{c}$ Abbreviations: $V_{\text {on }}=$ turn-on voltage at $1 \mathrm{~cd} \mathrm{~m}^{-2}$, $L_{\max }=$ maximum luminance, $\eta_{\mathrm{P}, \max }, \eta_{\mathrm{C}, \max }$ and $\eta_{\mathrm{ext} \text { max }}=$ maximum power, current and external efficiencies, respectively. CIE $=$ Commission International de l'Éclairage coordinates at $8 \mathrm{~V}$. 

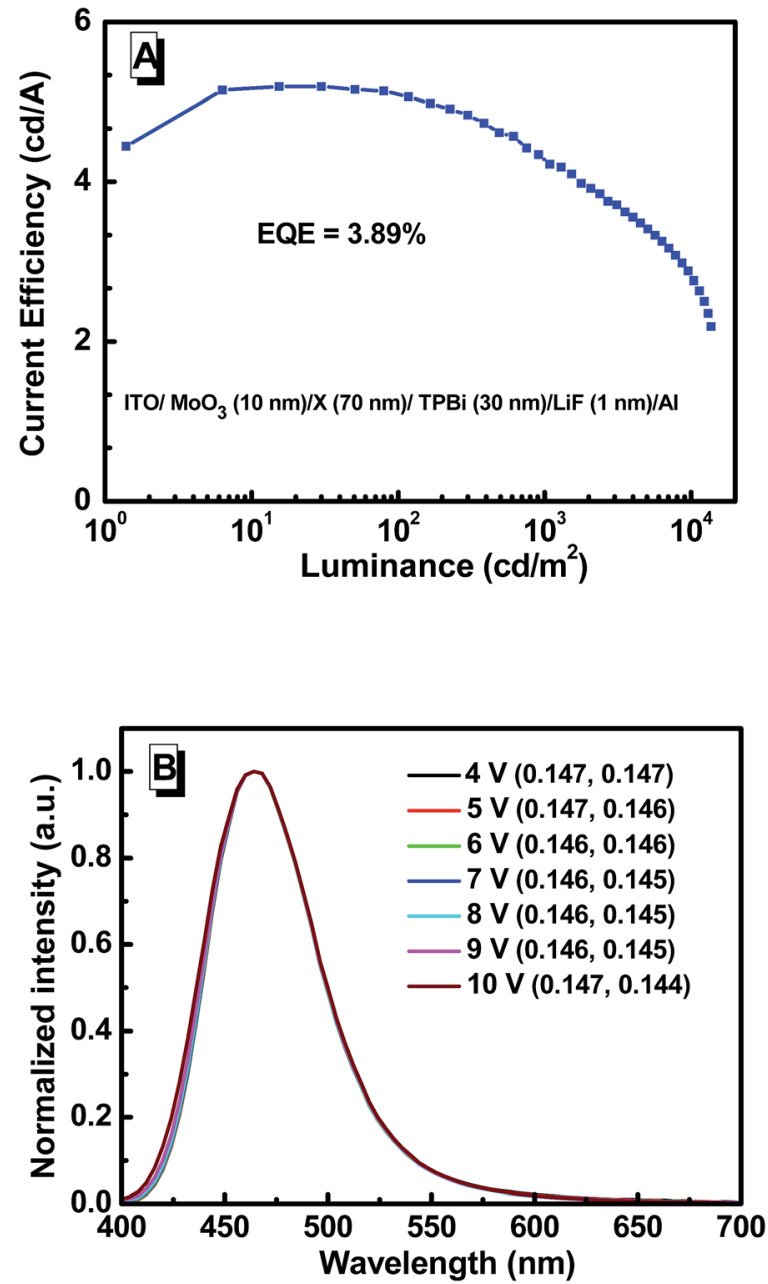

Fig. 8 (A) Current efficiency-luminance characteristics and (B) EL spectra of the device with a simple structure.

may probably break the charge balance in the EL device. Based on these results, we can draw the conclusion that 3TPA-CN is a promising material for deep-blue OLED devices with simple structure and high efficiency. ${ }^{9-12}$

Inspired by the good efficiency of 3TPA-CN as emitting layer in deep blue devices, further optimization was conducted. From the low temperature phosphorescence characteristics, the $E_{\mathrm{T}}$ value of 3TPA-CN was estimated to be $2.42 \mathrm{eV}$. To further confine excitons in the EML and enhance the device performance of 3TPA-CN-based devices, commercially available 1,3-bis(3,5dipyrid-3-yl-phenyl)benzene (BmPyPb) $\left(E_{\mathrm{T}}=2.70 \mathrm{eV}\right)$ was selected to be the host for the fabrication of doped devices. ${ }^{28}$ Also, an additional layer of $\mathrm{BmPyPb}$ was inserted to enhance the transfer of electrons. Devices with the configuration of ITO/ $\mathrm{MoO}_{3}(10 \mathrm{~nm}) / \mathrm{NPB}(60 \mathrm{~nm}) / \mathrm{mCP}(10 \mathrm{~nm}) / \mathrm{BmPyPb}: x \%$ 3TPA-CN $(20 \mathrm{~nm}) / \mathrm{BmPyPb}(10 \mathrm{~nm}) / \mathrm{TPBi}(35 \mathrm{~nm}) / \mathrm{LiF}(1 \mathrm{~nm}) / \mathrm{Al}$ were constructed (Fig. 9, S16 and S17, ESI $\dagger$ ). The emission wavelength and turn-on voltages are almost the same when the doped concentration was changed to $30 \%, 40 \%$ and $50 \%$. With the introduction of $\mathrm{BmPyPb}$, the turn-on voltage decreased to $3.6 \mathrm{~V}$, disclosing the easier transfer of carriers in the devices. At a doping concentration of $30 \%$, a CIE coordinate of $(0.15,0.17)$ is obtained with good stability. The $\mathrm{CIE}_{y}$ suffers a modest change when further increasing the doping concentration of 3TPA-CN. More interestingly, even lower than the results of device E (Table 2), the current efficiencies show an increase in comparison with that of the multi-layer nondoped device. At a lower doping concentration of $30 \%$, the current efficiency can be $5.04 \mathrm{~cd} \mathrm{~A}^{-1}$, then reach $5.07 \mathrm{~cd} \mathrm{~A}^{-1}$ when the concentration is increased to $40 \%$. However, when the concentration is further increased, the efficiency drops a little. On the whole, among the doped devices fabricated, device $\mathrm{F}$ (Table 2) employing 30\% doped concentration as the EML showed better deep-blue emission with a maximum EQE of $3.35 \%$, CE of $5.04 \mathrm{~cd} \mathrm{~A}^{-1}$ and PE of $5.02 \mathrm{~lm}$ $\mathrm{W}^{-1}$, meanwhile the maximum luminance is $7257 \mathrm{~cd} \mathrm{~m}^{-2}$. Even at a luminance of $1000 \mathrm{~cd} \mathrm{~m}^{-2}$, the $\mathrm{CE}$ of the device still remained at $3.27 \mathrm{~cd} \mathrm{~A}^{-1}$, demonstrating a relatively low efficiency roll-off among doped devices. Even though the performance is not higher than that of device E, 3TPA-CN is also a good candidate for guest material in deep-blue OLEDs.

\section{Characterization of phosphorescent OLEDs using 3TPA-CN as host}

From the above results, 3TPA-CN can be used as emitting layer and guest material with high performance. Due to the block effect towards non-radiative path, the utilization of AIE materials as hosts can contribute to efficient emissions. ${ }^{30}$ Considering its characteristics of persistence towards aggregation and high quantum yield, how about utilizing 3TPA-CN as host material instead of its general role as emissive layers? With this question in mind, after considering the possible energy matching, devices with a configuration of $\mathrm{ITO} / \mathrm{MoO}_{3} / \mathrm{NPB}$ $(60 \mathrm{~nm}) / \mathrm{mCP} \quad(10 \mathrm{~nm}) /$ host:PO-01 $(20 \mathrm{~nm}, 10 \mathrm{wt} \%) / \mathrm{TPBI}$ $(40 \mathrm{~nm}) / \mathrm{LiF}(1 \mathrm{~nm}) / \mathrm{Al}$ were fabricated, in which the host material was 3TPA-CN. For comparison, the devices with CBP as host were also fabricated (Fig. 9B). The transfer of energy and charge from the AEE host to the dopant successfully occurs, as shown in Fig. 10, the $V_{\text {on }}$ can be decreased to as low as $2.8 \mathrm{~V}$ by using 3TPA-CN as the host instead of CBP (3.2 V). Although the emission wavelength showed a small red-shift to $564 \mathrm{~nm}$, the CIE values can be stabilized at $(0.50,0.50)$. Device I with 3TPACN as the host achieves better EL performance, with the maximum current and power efficiency of $57.4 \mathrm{~cd} \mathrm{~A}^{-1}$ and $52.0 \mathrm{~lm} \mathrm{~W}^{-1}$, respectively, and a corresponding maximum EQE of $18.2 \%$. It is worth noting that even at a luminance of 10000 $\mathrm{cd} \mathrm{m}^{-2}$, the CE and EQE of the device can still remain $52.6 \mathrm{~cd}$ $\mathrm{A}^{-1}$, and $16.8 \%$, respectively, demonstrating a relatively low efficiency roll-off. The device performance can be comparable to the results of excellent orange emission devices reported in the literature. ${ }^{39,40}$ Under the same conditions, device J (Table 2) utilizing CBP as the host exhibits a maximum CE and EQE of $50.6 \mathrm{~cd} \mathrm{~A}^{-1}$ and $15.7 \%$, respectively. In comparison with the device using CBP as the host, the better performance of device I suggests that 3TPA-CN can be a good candidate for host material in orange PhOLEDs. Thus, this further indicates that the utilization of AEE materials as host materials in PhOLEDs might be a new choice in photonic and electronic research field. 


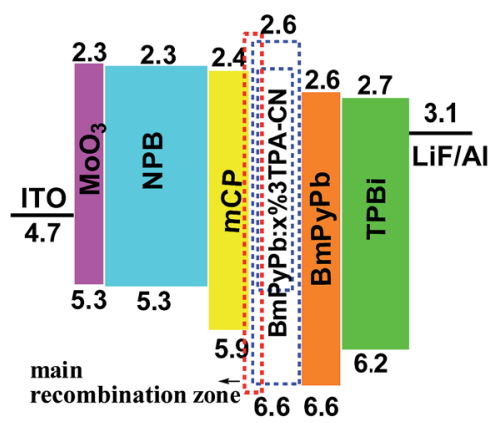

A

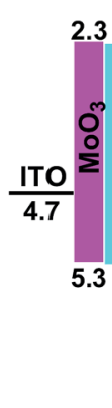

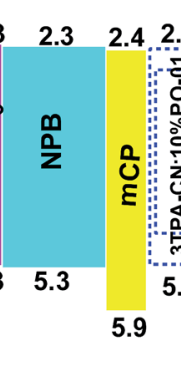

B

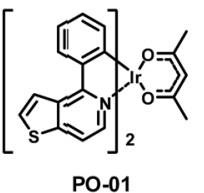<smiles>c1cncc(-c2cccc(-c3cc(-c4cccnc4)cc(-c4cccc(-c5cccnc5)c4)c3)c2)c1</smiles>

$\mathrm{BmPyPb}$

Fig. 9 Energy level diagram of the doped devices (A: 3TPA-CN as the guest, B: 3TPA-CN as the host).

\section{Conclusions}

In summary, through convenient synthetic routes, according to the design idea for AIEgens, four new benzene-cored luminogens were prepared with good thermal and photophysic stabilities. TPA-based luminogens show weak AEE effect while TPE-based ones exhibit typical AIE characteristics. Interestingly, TPA-based luminogens demonstrate blue-shifted emissions in films compared to those in solution. The fabricated devices show tunable deep-blue to green emissions. Due to its excellent hole transporting ability, without the additional hole-transporting layer, 3TPA-CN with a simple nondoped fluorescent device structure exhibited deep-blue emission and high performance, with a CE of $5.21 \mathrm{~cd} \mathrm{~A}^{-1}$, EQE of $3.89 \%$, and CIE coordinates of $(0.15,0.14)$. Excitingly, 3TPA-CN can serve as an excellent host for orange emissive PhOLEDs, with a maximum $\mathrm{CE}$ and EQE of $57.4 \mathrm{~cd} \mathrm{~A}^{-1}$ and $18.2 \%$, respectively, higher than those of CBP under the same conditions. All these results indicate that the rational decoration of benzene core is meaningful and promising for the design of excellent luminogens for
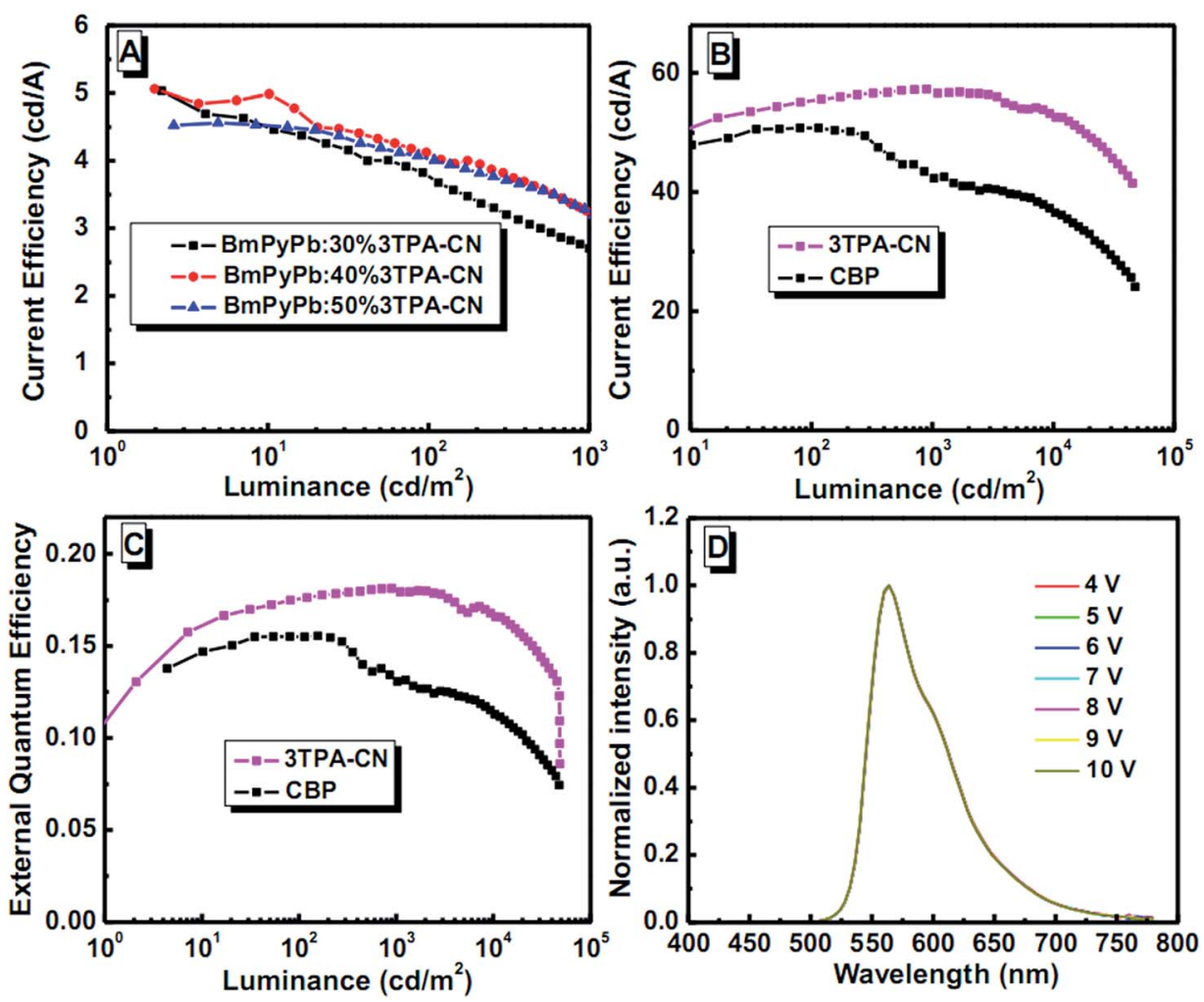

Fig. 10 (A) Change in current efficiency with the luminance in doped devices of 3TPA-CN with different doping concentrations (30\%, 40\% and $50 \%)$. Device configurations: ITO/MoO $3(10 \mathrm{~nm}) / \mathrm{NPB}(60 \mathrm{~nm}) / \mathrm{mCP}(10 \mathrm{~nm}) / \mathrm{BmPyPb}: x \% 3 \mathrm{TPA}-\mathrm{CN}(20 \mathrm{~nm}) / \mathrm{BmPyPb}(10 \mathrm{~nm}) / \mathrm{TPBi}(35 \mathrm{~nm}) / \mathrm{LiF}$ $(1 \mathrm{~nm}) /$ Al. All values are reported in wt\% of 3TPA-CN in BmPyPb. (B) Current efficiency-luminance characteristics, (C) external quantum efficiency-luminance characteristics and (D) EL spectra of the device with PO-01 as the guest. Device configurations: ITO/MoO $/ \mathrm{NPB}(60 \mathrm{~nm}) / \mathrm{mCP}$ $(10 \mathrm{~nm}) / 3 T P A-C N: P O-01(20 \mathrm{~nm}, 10 \mathrm{wt} \%) / \mathrm{TPBI}(40 \mathrm{~nm}) / \mathrm{LiF}(1 \mathrm{~nm}) / \mathrm{Al}$. 
OLED applications. Also, the successful utilization of 3TPA-CN as the host material in PhOLEDs might open up a new avenue for the practical application of AIEgens in the photonic and electronic research fields.

\section{Acknowledgements}

The authors are grateful to the National Fundamental Key Research Program (2013CB834701), and the National Science Foundation of China (no. 21325416, 51573140, 51333007 and 91433201) for financial support.

\section{Notes and references}

1 C. W. Tang and S. A. Vanslyke, Appl. Phys. Lett., 1987, 51, 913. 2 W. Wu, R. Tang, Q. Li and Z. Li, Chem. Soc. Rev., 2015, 44, 3997.

3 G. M. Farinola and R. Ragni, Chem. Soc. Rev., 2011, 40, 3467.

4 M. C. Gather, A. Kohnen and K. Meerholz, Adv. Mater., 2011, 23, 233.

5 H. Uoyama, K. Goushi, K. Shizu, H. Nomura and C. Adachi, Nature, 2012, 492, 234.

6 K. R. J. Thomas, M. Velusamy, J. Lin, Y. Tao and C. Chuen, Adv. Funct. Mater., 2004, 14, 387.

7 W. Jia, X. Feng, D. Bai, Z. Lu, S. Wang and G. Vamvounis, Chem. Mater., 2005, 17, 164.

8 L. Chen, Y. Jiang, H. Nie, P. Lu, H. H. Y. Sung, I. D. Williams, H. S. Kwok, F. Huang, A. Qin, Z. Zhao and B. Z. Tang, Adv. Funct. Mater., 2014, 24, 3621.

9 J. Y. Kim, T. Yasuda, Y. S. Yang and C. Adachi, Adv. Mater., 2013, 25, 2666.

10 W. Yuan, P. Lu, S. Chen, J. W. Y. Lam, Z. Wang, Y. Liu, H. S. Kwok, Y. Ma and B. Z. Tang, Adv. Mater., 2010, 22, 2159.

11 J. Huang, N. Sun, J. Yang, R. Tang, Q. Li, D. Ma and Z. Li, Adv. Funct. Mater., 2014, 24, 7645.

12 W. Qin, J. Liu, S. Chen, J. W. Y. Lam, M. Arseneault, Z. Yang, Q. Zhao, H. S. Kwok and B. Z. Tang, J. Mater. Chem. C, 2014, 2, 3756 .

13 T. J. Park, W. S. Jeon, J. J. Park, S. Y. Kim, Y. K. Lee, J. Jang, J. H. Kwon and R. Pode, Appl. Phys. Lett., 2008, 92, 113308.

14 C. H. Chien, C. K. Chen, F. M. Hsu, C. F. Shu, P. Chou and C. H. Lai, Adv. Funct. Mater., 2009, 19, 560.

15 X. Xing, L. Zhang, R. Liu, S. Li, B. Qu, Z. Chen, W. Sun, L. Xiao and Q. Gong, ACS Appl. Mater. Interfaces, 2012, 4, 2877.

16 J. Ye, Z. Chen, M. K. Fung, C. Zheng, X. Ou, X. Zhang, Y. Yuan and C. S. Lee, Chem. Mater., 2013, 25, 2630.

17 Y. Yuan, J. Chen, F. Lu, Q. Tong, Q. Yang, H. W. Mo, T. W. Ng, F. L. Wong, Z. Guo, J. Ye, Z. Chen, X. Zhang and C. S. Lee, Chem. Mater., 2013, 25, 4957.
18 J. Luo, Z. Xie, J. W. Y. Lam, L. Cheng, H. Chen, C. Qiu, H. S. Kwok, X. Zhan, Y. Liu, D. Zhu and B. Z. Tang, Chem. Commun., 2001, 1740.

19 L. Yao, S. Zhang, R. Wang, W. Li, F. Shen, B. Yang and Y. Ma, Angew. Chem., Int. Ed., 2014, 53, 2119.

20 Q. Li and Z. Li, Sci. China: Chem., 2015, 58, 1800.

21 X. Ji, P. Wang, H. Wang and F. Huang, Chin. J. Polym. Sci., 2015, 33, 890.

22 J. Mei, N. L. C. Leung, R. T. K. Kwok, J. W. Y. Lam and B. Z. Tang, Chem. Rev., 2015, 115, 11718.

23 J. Yang, J. Huang, Q. Li and Z. Li, J. Mater. Chem. C, 2016, DOI: 10.1039/c5tc03262h.

24 X. Tang, L. Yao, H. Liu, F. Shen, S. Zhang, H. Zhang, P. Lu and Y. Ma, Chem.-Eur. J., 2014, 20, 7589.

25 S. Liu, F. He, H. Wang, H. Xu, C. Wang, F. Li and Y. Ma, J. Mater. Chem. C, 2008, 18, 4802.

26 J. Huang, N. Sun, Y. Dong, R. Tang, P. Lu, P. Cai, Q. Li, D. Ma, J. Qin and Z. Li, Adv. Funct. Mater., 2013, 23, 2329.

27 J. Huang, N. Sun, P. Chen, R. Tang, Q. Li, D. Ma and Z. Li, Chem. Commun., 2014, 50, 2136.

28 X. Zhan, N. Sun, Z. Wu, J. Tu, L. Yuan, X. Tang, Y. Xie, Q. Peng, Y. Dong, Q. Li, D. Ma and Z. Li, Chem. Mater., 2015, 27, 1847.

29 X. Zhan, Z. Wu, Y. Lin, S. Tang, J. Yang, J. Hu, Q. Peng, D. Ma, Q. Li and Z. Li, J. Mater. Chem. C, 2015, 3, 5903.

30 J. Huang, X. Yang, X. Li, P. Chen, R. Tang, F. Li, P. Lu, Y. Ma, L. Wang, J. Qin, Q. Li and Z. Li, Chem. Commun., 2012, 48, 9586.

31 T. H. Huang, J. T. Lin, L. Y. Chen, Y. T. Lin and C. C. Wu, Adv. Mater., 2006, 18, 602.

32 J. Huang, N. Sun, J. Yang, R. Tang, Q. Li, D. Ma, J. Qin and Z. Li, J. Mater. Chem., 2012, 22, 12001.

33 Q. Zhang, J. Li, K. Shizu, S. Huang, S. Hirata, H. Miyazaki and C. Adachi, J. Am. Chem. Soc., 2012, 134, 14706.

34 M. Taneda, K. Shizu, H. Tanaka and C. Adachi, Chem. Commun., 2015, 51, 5028.

35 S. L. Lin, L. H. Chan, R. H. Lee, M. Y. Yen, W. J. Kuo, C. T. Chen and R. J. Jeng, Adv. Mater., 2008, 20, 3947.

36 J. S. Swensen, E. Polikarpov, A. V. Ruden, L. Wang, L. S. Sapochak and A. B. Padmaperuma, Adv. Funct. Mater., 2011, 21, 3250.

37 S. L. Lai, W. Y. Tong, S. C. F. kui, M. Y. Chan, C. C. Kwok and C. M. Che, Adv. Funct. Mater., 2013, 23, 5168.

38 S. Chen, Q. Wu, M. Kong, X. Zhao, Z. Yu, P. Jia and W. Huang, J. Mater. Chem. C, 2013, 1, 3508.

39 S. Lee, K. H. Kim, D. Limbach, Y. S. Park and J. J. Kim, Adv. Funct. Mater., 2013, 23, 4105.

40 D. Zhang, L. Duan, Y. Li, H. Li, Z. Bin, D. Zhang, J. Qiao, G. Dong, L. Wang and Y. Qiu, Adv. Funct. Mater., 2014, 24, 3551. 